\title{
How the FMR1 gene became relevant to female fertility and reproductive medicine
}

\author{
Norbert Gleicher ${ }^{1,2}$ *, Vitaly A. Kushnir ${ }^{1}$, Andrea Weghofer ${ }^{1,3}$ and David H. Barad ${ }^{1,2}$ \\ 1 Center for Human Reproduction, New York, NY, USA \\ 2 Foundation for Reproductive Medicine, New York, NY, USA \\ ${ }^{3}$ Department of Obstetrics and Gynecology, Medical University Vienna, Vienna, Austria
}

\section{Edited by:}

Lisa M. Pastore, University of Virginia, USA

\section{Reviewed by:}

Alex Vincent Postma, Academic

Medical Center, Netherlands

Dale William Stovall, Riverside Health

System, USA

\section{*Correspondence:}

Norbert Gleicher, Center for Human Reproduction, 21 East 69th Street,

New York, NY, USA

e-mail:ngleicher@thechr.com
This manuscript describes the 6 year evolution of our center's research into ovarian functions of the FMR1 gene, which led to the identification of a new normal CGGn range of 26-34. This "new" normal range, in turn, led to definitions of different alleles (haplotypes) based on whether no, one or both alleles are within range. Specific alleles then were demonstrated to represent distinct ovarian aging patterns, suggesting an important FMR1 function in follicle recruitment and ovarian depletion of follicles. So called low alleles, characterized by $\mathrm{CGGn}_{<26}$, appear associated with most significant negative effects on reproductive success. Those include occult primary ovarian insufficiency (OPOI), characterized by prematurely elevated follicle stimulating hormone (FSH) and prematurely low anti-Müllerian hormone, and significantly reduced clinical pregnancy rates in association with in vitro fertilization (IVF) in comparison to women with normal (norm) and high $\left(C G G n_{>34}\right)$ alleles. Because low FMR1 alleles present in approximately $25 \%$ of all females, FMR1 testing at young ages may offer an opportunity for earlier diagnosis of OPOI than current practice allows. Earlier diagnosis of OPOI, in turn, would give young women the options of reassessing their reproductive schedules and/or pursue fertility preservation via oocyte cryopreservation when most effective.

Keywords: FMR1 gene, FSH, AMH, follicle maturation, fertility preservation
It has been known for decades that premutation range mutations of the fragile $\mathrm{X}$ mental retardation $(F M R 1)$ gene $\left(\mathrm{CGGn}_{55-200}\right)$ are associated with greatly increased female risk of primary ovarian insufficiency (POI; Wittenberger et al., 2007). Neither endocrinologists nor geneticists, however, considered the possibility that the gene, beyond widely investigated neuro-psychiatric effects, giving it the name "fragile X chromosome," may also have a role in ovarian function.

This idea arose at our center in 2008, and was immediately encouraged when we demonstrated that CGGn appeared in infertile women associated with follicle stimulating hormone (FSH) and anti-Müllerian hormone $(\mathrm{AMH})$, both parameters of functional ovarian reserve (FOR; Gleicher etal., 2009a). Moreover, women with autoimmune-associated occult POI (OPOI, also called premature ovarian aging, POA) demonstrated significantly lower CGGn (in the new "normal" range) than non-autoimmune women with OPOI who, therefore, were presumed to have FMR1-related OPOI (Gleicher et al., 2009b).

The hypothesis of an FMR1-associated ovarian function effect was further supported when a literature review revealed a 1991 article by $\mathrm{Fu}$ etal. (1991) which, as a side note, in the general population reported a very prominent distribution peak at $\mathrm{CGGn}_{30-31}$ (Figure 1). It to us appeared "destined" to represent an additional function of the FMR1 gene within what then was considered the normal range of CGGn. Seeing this spiking distribution peak in an otherwise spread-out distribution pattern, we suspected it to represent the gene's hitherto unknown ovarian function.

We subsequently found a 2003 manuscript by Chen et al. (2003) reporting $\mathrm{CGGn}_{30}$ as the switching point between positive and negative message and maximal translation for the gene, which only further strengthened our conviction that we, likely, indeed had discovered a new ovarian function of the FMR1 gene. It, however, remained to be determined what exactly this function entailed.

\section{DEFINING THE OVARIAN FUNCTION OF THE FMR1 GENE}

We then in a series of papers attempted to define how the FMR1 gene affects ovarian function. First, we established the "normal" CGGn range for this presumed ovarian function of the gene at 26-34 (Gleicher et al., 2010c,d), a range that not only included at midpoint above noted switching point between positive and negative message at $\mathrm{CGGn}_{30}$ (Chen et al., 2003) but also the large distribution peak at $\mathrm{CGGn}_{29-30}$, reported by Fu etal. (1991; Figure 1).

Establishing a new "normal" range allowed for definition of new alleles (haplotypes) for the gene, which, of course, were distinctively different from the traditional CGGn mutations [normal, intermediate ("gray zone"), premutation and full mutation], used to define neuro-psychiatric risks, including the fragile $\mathrm{X}$ syndrome (Wittenberger et al., 2007). These new alleles (haplotypes) were also called genotypes, and were defined as normal (norm) if both alleles were in normal range, as heterozygous 


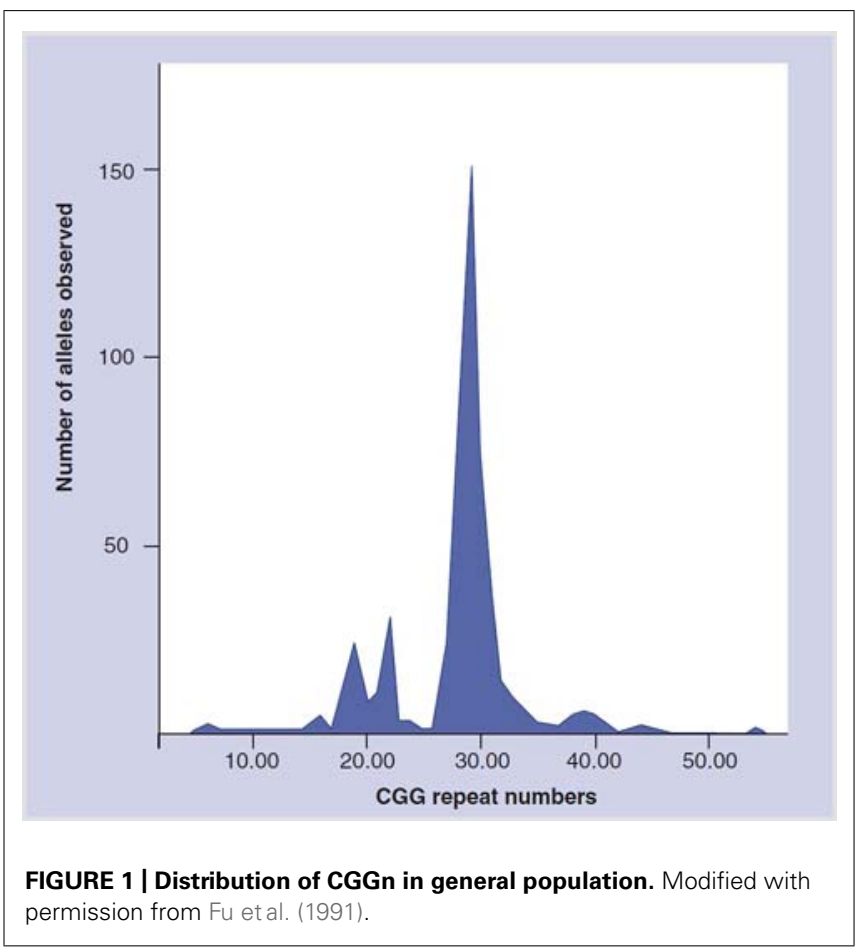

(het) if one allele was outside normal range and as homozygous (hom) if both alleles were outside normal range (Gleicher et al., 2010c,d).

Up to that point, the genetic literature, almost exclusively, had only concentrated on the expansive properties of the FMR1 gene into abnormally high CGGn ranges. They were associated with well known neuro-psychiatric conditions at premutation (CGGn $\sim 55-200$ ) and full mutation range CGGn expansions $\left(\mathrm{CGGn}_{>} 200\right)$. Our center's research, therefore, initially also concentrated only on expanding CGGn to the right of the by $\mathrm{Fu}$ etal. (1991) reported population peak at $\mathrm{CGGn}_{29-30}$ (Gleicher et al., 2009c,d). Because of the rather symmetrical distribution pattern of CGGn on both sides of the distribution peak (Figure 1), we, however, also initiated investigations of low alleles $\left(\mathrm{CGGn}_{<26}\right)$, once we discovered that the risk for OPOI on both sides of the CGGn distribution peak was similar (Gleicher et al., 2009e).

As further discussed below, we quickly learned that low CGGn counts, indeed, appear associated with some of the most important effects of the FMR1 gene on female reproduction. We, therefore, started subdividing above described "new" haplotypes (genotypes) into so-called sub-genotypes or low $\left(\mathrm{CGGn}_{<26}\right)$ and high $\left(\mathrm{CGGn}_{>34}\right)$ alleles (Gleicher et al., 2010c,d).

Assessing young oocyte donors and infertile women at different ages, using these newly defined FMR1 alleles, ovarian functions of the gene came into clearer view: different FMR1 alleles were found associated with fairly typical ovarian aging patterns. Women with norm genotypes based on AMH levels followed a more or less normal ovarian aging pattern. Het-low carriers, in contrast, lost FOR at an accelerated pace, while carriers of het-high alleles throughout life appeared to recruit slower than other haplotypes and, therefore, at advanced ages present with best FOR (Gleicher and Barad, 2010; Gleicher et al., 2010c, 2012b,c).

Because hom patients are rare, especially when sub-divided into sub-genotypes, functions of hom haplotypes are not as well defined as norm and het patients. Preliminary, and mostly yet unpublished observations of hom populations suggest typical accelerated behavior if both alleles are either high or low but high/low hom patients appear to differ very significantly in ovarian phenotype, likely based on which allele is inactivated (Gleicher et al., unpublished data).

Based on these clinical observations we, therefore, concluded that the FMR1 gene, likely, is involved in follicle recruitment, though in which way still, remains to be determined.

Additional observations added to the picture: while the normal range of $\mathrm{CGGn}_{26-34}$ is the same among all races, the distribution of individual alleles varies between races, with women of African descent demonstrating disproportionally more low and fewer high alleles, while Asian women (most were Chinese Han) demonstrate disproportionally more high FMR 1 and very few low alleles. Caucasians (including Hispanics), most diverse in FMR1 allele distribution, fell in between the other two racial groups (Gleicher et al., 2010b, 2012a).

An increased prevalence of low alleles in women of African descent may, at least in part, contribute to their lower IVF pregnancy rates in comparison to Caucasian women, widely reported in the IVF literature (Gleicher et al., 2011). Women with low FMR1 alleles, adjusted for covariates such as age, demonstrate significantly lower IVF pregnancy rates than women with norm genotypes (Gleicher et al., 2010d, 2011), an observation recently again confirmed in a greatly expanded study (Kushnir et al., 2014).

Though clinically often difficult to recognize, differences in ovarian aging patterns based on FMR1 mutations can already be observed in young oocyte donors (Gleicher et al., 2013a) and, therefore, can also be utilized to optimize egg donor selection (Gleicher et al., 2010a). A 4 year follow up of young, carefully selected egg donors recently demonstrated that donors carrying low FMR1 alleles already at their young ages significantly deviated from donors with norm and high alleles in FOR, as assessed by AMH levels (Kushnir et al., unpublished data).

Trying to better understand underlying physiological mechanisms for the observed IVF outcome differences between FMR1 alleles, we discovered that women with low alleles convert dehydroepiandrosterone (DHEA) to testosterone $(\mathrm{T})$ poorer than other mutations (Weghofer et al., 2012). Since LFOR is associated with low T levels (Gleicher et al., 2013b) and the increase in T after DHEA supplementation is predictive of pregnancy success in IVF (Gleicher et al., 2013c), this observation, at least partially, may explain lower IVF pregnancy rates in association with low FMR1 alleles.

In over 5 years we, thus, succeeded in describing a well defined new function of the FMR1 gene, which affects the ovarian aging process, reflected in FOR levels at different ages. Key studies by other investigators are briefly summarized below.

\section{CONCOMITANT OBSERVATIONS IN OTHER LABORATORIES}

In a mouse model that carries a human FMR1 premutation allele, Lu etal. (2012) reported that FMR1 premutation RNA 
reduces the number of growing follicles in ovaries, and, thereby, impairs female fertility, possibly acting through the Akt/mTOR pathway. In another mouse model, a FX-PM mouse with 130 CGG repeats, Hoffman et al. (2012) demonstrated normal ovarian development and establishment of a normal primordial follicle pool. The animals, however, demonstrated much faster follicle loss, in concordance with what could be interpreted as the equivalent of OPOI (Hoffman etal., 2012). In an even more recent paper, Ascano etal. (2012) reported that a Fmr1-/mouse model showed unexpected signs of premature follicular over-development. All of these animal studies, therefore, offer supporting evidence for a significant role of the FMR1 gene in ovarian physiology.

Ferder et al. (2013) demonstrated in a rat model the expression of fragile $\mathrm{X}$ mental retardation protein (FMRP) in granulosa, theca and germ cells at all stages of follicle development. Moreover, these authors also demonstrated changes in Fmr1 expression at protein as well as mRNA levels. Specifically, FMRP expression increased with advancing follicle development, with preantral and early antral follicles demonstrating similar Fmr1 transcript, and decreased expression in preovulatory transcripts. The authors detected four different isoforms of FMRP during different stages of follicle maturation, with expression patterns varying from what they observed in brain and testis (Ferder et al., 2013).

Demonstration of similarly broad presence of FMR1 at mRNA and protein levels in the human ovary would resolve further questions about the importance of this gene for ovarian physiology. Our center is currently conducting studies to further elucidate the relevance of FMR1 mRNA and FMRP in different clinical situations affecting ovarian function.

Published clinical data from outside our laboratory have so far not included information on low CGGn alleles and, therefore, appear limited in scope and clinical relevance. Some, however, do offer interesting information: Schuettler et al. (2011), who assessed DNA and RNA samples from 74 women with idiopathic POI in order to evaluate the quantitative expression of FMR1 in peripheral leukocytes and in relation to their CGGn is one example. Only women with POI demonstrated a large variance in FMR1 transcript from leukocyte RNA samples, but this variance did not correlate to CGGn. They concluded that all women with $\mathrm{CGGn}_{<26}$ and/or $\mathrm{CGGn}_{>34}$, in other words with CGGn outside of the new "normal" range for ovarian function we had described, appear to experience what they called "relaxed" transcription control. As a consequence, they, therefore, are at risk for OPOI (i.e., premature ovarian aging).

Pastore etal. (2012) reported that women with LFOR present with overrepresentation of $\mathrm{CGGn}_{35-44}$ alleles, which in our mutation classification would mostly correspond to high FMR1 alleles (Gleicher et al., 2010c,d). Similarly, Barasoain etal. (2013) reported in a Basque population with FSH levels above $10.0 \mathrm{mIU} / \mathrm{mL}$, using traditional mutation designations for neuro-psychiatric risks, an increased prevalence of intermediate and premutation range alleles. These authors, therefore, also only commented on what we would describe as high allele patients.
But not all reported studied were able to demonstrate differences in CGGn between fertile and infertile women. De Geyter et al. (2014) were unable to demonstrate "expanded" CGGn in infertile women and Voorhuis et al. (2013), in our opinion incorrectly, dismissed a role for the FMR1 gene in the ovarian aging process because CGGn was in their study not predictive of age at menopause.

While we never specifically investigated in our studies whether the FMR1 gene affects age of menopause, we also noted that FOR curves for all the different FMR1 alleles unite at advanced ages, suggesting a common menopause age for all of the haplotypes we have described. Different haplotypes, however, "take different roads" to that meeting point (Gleicher et al., unpublished data). We, therefore, do not agree with the conclusions reached by Voorhuis et al. (2013).

Furthermore, some reports do suggest a possible FMR1 effect on menopause age: Sullivan et al. (2005) and Ennis et al. (2006) suggest such an effect likely around $\mathrm{CGGn}_{<80}$, a midrange expansion size within the premutation range, concluding that menopause age is associated in a non-linear way with CGGn.

\section{THE FUTURE}

Elucidating the function of FMR1 mRNA and FMRP at different stages of follicle maturation appears of primary importance. Based on above described clinical observation we, indeed, would suspect that both may differ in association with different FMR1 alleles (genotypes and sub-genotypes).

The FMR1 gene may, however, also acquire additional significance as a prognostic diagnostic tool: we above noted that our data in young oocyte donors suggest that FOR in young women with low FMR1 mutations already at very young ages declines significantly more rapidly than in women with either norm or hom alleles. Since approximately a quarter of all women carry a low FMR1 allele (Gleicher et al., 2010c,d), this observation would suggest that approximately one quarter of the female population can be defined as "at risk" for LFOR at prematurely young ages.

If confirmed, assessments of FMR1 mutations at young ages, possibly in association with other risk factors, could identify a high risk sub-population of young women, who, with careful longitudinal assessments of FOR, can be diagnosed at much younger ages than current practice allows for. Such early diagnosis of LFOR would then allow women to either enhance their reproductive timing or pursue fertility preservation at still young ages, when fertility preservation is most effective.

\section{REFERENCES}

Ascano, M. Jr., Mukjerjee, N., Bandaru, P., Miller, J. B., Nusbaum, J., Corcoran, D. L., et al. (2012). FMR1 targets distinct mRNA sequence element to regulate protein expression. Nature 492, 382-386. doi: 10.1038/nature11737

Barasoain, M., Barrenetxea, G., Huerta, I., Télez, M., Cérez, C., Criado, B., et al. (2013). Study of FMR1 gene association with ovarian dysfunction in a sample from the Basque Country. Gene 521, 145-149. doi: 10.1016/j.gene.2013.03.032

Chen, L. S., Tassone, F., Sahota, P., and Hagerman, P. J. (2003). The (CGG) ${ }_{n}$ repeat element within the $5^{\prime}$ untranslated region of the FMR1 message provides both positive and negative cis effects on in vivo translation of a downstream reporter. Hum. Mol. Genet. 12, 3067-3074. doi: 10.1093/hmg/ddg331 
De Geyter, C., M'rabel, N., de Geyter, J., Zürcher, S., Moffat, R., Bösch, N., et al. (2014). Similar prevalence of expanded CGG repeat lengths in the fragile X mental retardation 1 gene among infertile women and among women with proven fertility: a prospective study. Genet. Med. 16, 374-378. doi: 10.1038/gim.2013.146

Ennis, S., Ward, D., and Murray, A. (2006). Nonlinear association between CGG repeat number and age of menopause in FMR1 premutation carriers. Eur. J. Hum Genet. 14, 253-255. doi: 10.1038/sj.ejhg.5201510

Ferder, I., Parborell, F., Sundblad, V., Chiauzzi, V., Gómez, K., Charreau, E. H., et al. (2013). Expression of fragile X mental retardation protein and Fmr $1 \mathrm{mRNA}$ during folliculogenesis in the rat. Reproduction 145, 335-343. doi: 10.1530/REP12-0305

Fu, Y. H., Kuhl, D. P., Pizzuti, A., Pieretti, M., Sutcliffe, J. S., Richards, S., et al. (1991). Variations in CGG repeat of the fragile X site results in genetic instability: resolution of the Sherman paradox. Cell 67, 1047-1058. doi: 10.1016/0092-8674(91)90283-5

Gleicher, N., and Barad, D. H. (2010). Can the FMR1 gene predict early aging? Womens Health (Lond. Engl.) 6, 165-169. doi: 10.2217/whe.09.85

Gleicher, N., Kim, A., Barad, D. H., Shohat-Tal, A., Lazzaroni, E., Michaeli, T., etal. (2013a). FMR1-dependent variability of ovarian aging patterns is already apparent in young oocyte donors. Reprod. Biol. Endocrinol. 11, 80 doi: 10.1186/1477-7827-11-80

Gleicher, N., Kim, A., Weghofer, A., Kushnir, V. A., Shohat-Tal, A., Lazzaroni, E., et al. (2013b). Hypoandrogenism in association with diminished functional ovarian reserve. Hum. Reprod. 28, 1084-1091. doi: 10.1093/humrep/det033

Gleicher, N., Kim, A., Weghofer, A., Shohat-Tal, A., Lazzaroni, E., Lee, H. J., et al (2013c). Starting and resulting testosterone levels after androgen supplementation determine at all ages in vitro fertilization (IVF) pregnancy rates in women with diminished ovarian reserve (DOR). J. Assist. Reprod. Genet. 30, 49-62 doi 10.1007/s10815-012-9890-z

Gleicher, N., Kim, A., Weghofer, A., and Barad, D. H. (2012a). Differences in ovarian aging patterns between races are associated with ovarian genotypes and subgenotypes of the FMR1 gene. Reprod. Biol. Endocrinol. 10, 77. doi: 10.1186/14777827-10-77

Gleicher, N., Weghofer, A., Kim, A., and Barad, D. H. (2012b). Comparison of ovarian FMR1 genotypes and sub-genotypes in oocyte donors and infertile women. J. Assist. Reprod. Genet. 29, 529-532. doi: 10.1007/s10815-012-9745-7

Gleicher, N., Weghofer, A., Kim, A., and Barad, D. H. (2012c). The impact in older women of ovarian FMR1 genotypes and sub-genotypes on ovarian reserve. PLoS ONE 7:e33638. doi: 10.1371/journal.pone.0033638

Gleicher, N., Weghofer, A., and Barad, D. H. (2009a). A pilot study of premature ovarian senescence: I. Correlataion of triple CGG repeats on the FMR1 gene to ovarian reserve parameters FSH and anti-Müllerian hormone. Fertil. Steril. 91 1700-1706. doi: 10.1016/j.fertnstert.2008.01.098

Gleicher, N., Weghofer, A., and Barad, D. H. (2009b). A pilot study of premature ovarian senescence: II. Different genotype and phenotype for genetic and autoimmune etiologies. Fertil. Steril. 91, 1707-1011. doi: 10.1016/j.fertnstert.2008.01.099

Gleicher, N., Weghofer, A., Oktay, K., and Barad, D. H. (2009c). Can the FMR (fragile $\mathrm{X}$ ) gene serve as a predictor of response to ovarian stimulation? Reprod. Sci. 16, 462-467. doi: 10.1177/1933719108328617

Gleicher, N., Weghofer, A., Oktay, K., and Barad, D. H. (2009d). Correlation of triple repeats on the FMR1 (fragile X0 gene to ovarian reserve: a new infertility test? Acta Obstet. Gynecol. Scand. 88, 1024-1030. doi: 10.1080/000163409031 71058

Gleicher, N., Weghofer, A., Oktay, K., and Barad, D. (2009e). Relevance of triple CGG repeats in the FMR1 gene to ovarian reserve. Reprod. Biomed. Online 19, 385-390. doi: 10.1016/S1472-6483(10)60173-3

Gleicher, N., Weghofer, A., and Barad, D. H. (2010a). Can egg donor selection be improved? - A pilot study. Reprod. Biol. Endocrinol. 8, 76. doi: 10.1186/14777827-8-76
Gleicher, N., Weghofer, A., and Barad, D. H. (2010b). Effects of race/ethnicity on triple CGG counts in the FMR1 gene in infertile women and egg donors. Reprod. Biomed. Online 20, 485-491. doi: 10.1016/j.rbmo.2009.12.017

Gleicher, N., Weghofer, A., and Barad, D. H. (2010c). Ovarian reserve determinations suggest new function of FMR1 (fragile $\mathrm{X}$ gene) in regulating ovarian ageing. Reprod. Biomed. Online 20, 768-775. doi: 10.1016/j.rbmo.2010.02.020

Gleicher, N., Weghofer, A., Lee, I. H., and Barad, D. H. (2010d). FMR1 genotype with autoimmunity-associated polycystic ovary-like phenotype and decreased pregnancy chance. PLoS ONE 5:e15303. doi: 10.1371/journal.pone.0015303

Gleicher, N., Weghofer, A., Lee, I. H., and Barad, D. H. (2011). Association of FMR1 genotypes with in vitro fertilization (IVF) outcomes based on ethnicity/race. PLoS ONE 6:e18781. doi: 10.1371/journal.pone.0018781

Hoffman, G. E., Le, W. W., Entezam, A., Otsuka, N., Tong, Z. B., Nelson, L., et al. (2012). Ovarian abnormalities in mouse model of fragile X primary ovarian insufficiency. J. Histochem. Cytochem. 60, 439-456. doi: 10.1369/0022155412441002

Kushnir, V. A., Yu, Y., Barad, D. H., Weghofer, A., Himaya, E., Lee, H.-J., et al. (2014). Utilizing FMR1 mutations as predictors of treatment success in human in vitro fertilization. PLoS ONE 9:e102274. doi: 10.1371/journal.pone.0102274

Lu, C., Lin, L., Tan, H., Wu, H., Sherman, S. L., Gao, F., et al. (2012). premutation RNA is sufficient to cause primary ovarian insufficiency in mice. Hum. Molec. Genet. 21, 5039-5047. doi: 10.1093/hmg/dds348

Pastore, L. M., Young, S. L., Baker, V. L., Karns, L. B., Williams, C. D., and Silverman, L. M. (2012). Elevated prevalence of 35-44 FMR1 trinucleotide repeats in women with diminished ovarian reserve. Reprod. Sci. 19, 1226-1231. doi: $10.1177 / 1933719112446074$

Schuettler, J., Peng, Z., Zimmer, J., Sinn, P., von Hagens, C., Strwitzki, T., et al. (2011). Variable expression of the Fragile X Mental Retardation 1 (FMR1) gene in patients with premature ovarian failure syndrome is not dependent on number of (CGG)n triples in exon 1. Hum. Reprod. 26, 1241-1251. doi: 10.1093/humrep/der018

Sullivan, A. K., Marcus, M., Epstein, M. P., Allen, E. G., Anido, A. E., Paquin, J. J., et al. (2005). Association of FMR1 repeat size with ovarian dysfunction. Hum. Reprod. 20, 402-412. doi: 10.1093/humrep/deh635

Voorhuis, M., Onland-Moret, N. C., Fauser, B. C., Ploos van Amstel, H. K., van der Schouw, Y. T., and Broekmans, F. J. (2013). The association of CGG repeats in the FMR1 gene and timing of natural menopause. Hum. Reprod. 28, 496-501. doi: 10.1093/humrep/des392

Weghofer, A., Kim, A., Barad, D. H., and Gleicher, N. (2012). The impact of androgen metabolism and FMR1 genotypes on pregnancy potential in women with dehydroepiandrosterone (DHEA) supplementation. Hum. Reprod. 27, 3287-3293. doi: 10.1093/humrep/des265

Wittenberger, M. D., Hagerman, R. J., Sherman, S. L., McConkie-Rosell, A., Welt, C. K., Rebar, R. W., et al. (2007). The FMR1 premutation and reproduction. Fertil. Steril. 87, 456-465. doi: 10.1016/j.fertnstert.2006.09.004

Conflict of Interest Statement: David H. Barad and Norbert Gleicher are co-inventors of a number of pending patents, claiming diagnostic benefits from evaluations of CGGn on the FMR1 gene.

Received: 12 May 2014; accepted: 01 August 2014; published online: 29 August 2014. Citation: Gleicher N, Kushnir VA, Weghofer A and Barad DH (2014) How the FMR1 gene became relevant to female fertility and reproductive medicine. Front. Genet. 5:284. doi: 10.3389/fgene.2014.00284

This article was submitted to Genetic Disorders, a section of the journal Frontiers in Genetics.

Copyright (c) 2014 Gleicher, Kushnir, Weghofer and Barad. This is an open-access article distributed under the terms of the Creative Commons Attribution License (CC BY). The use, distribution or reproduction in other forums is permitted, provided the original author(s) or licensor are credited and that the original publication in this journal is cited, in accordance with accepted academic practice. No use, distribution or reproduction is permitted which does not comply with these terms. 